\title{
A Japanese patient with a mild Lenz-Majewski syndrome
}

\author{
Sumito Dateki - Tatsuro Kondoh · Gen Nishimura - Katsuaki Motomura • \\ Koh-ichiro Yoshiura $\cdot$ Akira Kinoshita $\cdot$ Hideo Kuniba Yoshiyuki Koga \\ Hiroyuki Moriuchi
}

Received: 10 April 2007/Accepted: 17 May 2007/Published online: 26 June 2007

(C) The Japan Society of Human Genetics and Springer 2007

\begin{abstract}
We report on a sclerosing bone dysplasia, associated with cutis laxa, enamel dysplasia, and mental retardation. The patient was a 17 -year-old Japanese boy of normal height and muscular build. Cutis laxa with prominent veins in the scalp and abdominal wall and delayed eruption of permanent teeth attracted the attention of clinicians in infancy and adolescence, respectively. The clinical manifestations included a progeroid facial appearance with prognathism, wrinkled skin, and interdigital webbing. The intelligence quotient was estimated at 60. Enamel dysplasia was histologically confirmed. Skeletal changes included calvarial hyperostosis, sclerosis of the skull base, an enlarged, sclerotic mandible, broad clavicles and ribs, and diaphyseal undermodeling of the tubular bones. Metaepiphyseal sclerosis or longitudinal striation was found in the long bones. Metaphyseal
\end{abstract}

S. Dateki $(\bowtie) \cdot$ T. Kondoh $\cdot$ K. Motomura

H. Moriuchi

Department of Pediatrics,

Nagasaki University Graduate School of Biomedical Sciences,

1-7-1, Sakamoto, Nagasaki 852-8501, Japan

e-mail:sum826@net.nagasaki-u.ac.jp

G. Nishimura

Department of Radiology, Tokyo Metropolitan Kiyose

Children's Hospital, Tokyo, Japan

K. Yoshiura $\cdot$ A. Kinoshita $\cdot$ H. Kuniba

Department of Human Genetics,

Nagasaki University Graduate School of

Biomedical Sciences, Nagasaki, Japan

Y. Koga

Division of Oral Pathology and Bone Metabolism,

Department of Developmental and Reconstructive Medicine,

Nagasaki University Graduate School of Biomedical Sciences,

Nagasaki, Japan equivalents of the axial skeleton showed dense osteosclerosis. These clinical and radiological manifestations overlapped with those of Lenz-Majewski syndrome. Unlike the classical phenotype of the disorder, however, he did not show brachymesophalangy with proximal symphalangism or growth failure. The present case may be considered to fall in the mildest end in the phenotypic continuum of Lenz-Majewski syndrome, suggesting that the clinical spectrum of the disorder may be broader than currently thought.

Keywords Cranial sclerosis - Cutis laxa ·

Lenz-Majewski syndrome $\cdot$ Mental retardation $\cdot$ Progeria

\section{Introduction}

Lenz-Majewski syndrome (LMS) (OMIM 151050) is a rare sclerosing bone dysplasia, first described by Braham (1969) in 1969 and subsequently named Lenz-Majewski hyperostotic dwarfism (Lenz and Majewski 1974; Robinow et al. 1977). Only nine affected individuals have been reported to date, and all were sporadic (Braham 1969; Macpherson 1974; Kaye et al. 1974; Lenz and Majewski 1974; Robinow et al. 1977; Gorlin and Whitley 1983; Chrzanowska et al. 1989; Nishimura et al. 1997; Saraiva 2000; Wattanasirichaigoon et al. 2004). The skeletal changes include progressive hyperostosis of the craniofacial bones with delayed closure of the fontanelles, diaphyseal cortical thickening of the tubular bones, and brachymesophalangy with proximal symphalangism. In addition, affected individuals show moderate to severe mental retardation, severe growth failure, cutis laxa, enamel hypoplasia, choanal atresia, nasolacrimal duct obstruction, and webbed fingers and toes. The prognosis is 
guarded. Most patients that have been reporteddied during childhood (Braham 1969; Macpherson 1974; Kaye et al. 1974; Majewski 2000). We report here on a Japanese boy whose manifestations can be considered to be a mild variant of LMS. Our experience raises the suspicion that the phenotypic range of LMS is broader than currently believed.

\section{Clinical report}

The patient was a 17-year-old Japanese boy born to nonconsanguineous healthy parents who were respectively 25 (mother) and 30 (father) years old at his birth. The family history was unremarkable. He was vaginally delivered at 40 weeks of gestation after an uneventful pregnancy. Apgar scores at 1 and 5 min were 8 and 10, respectively. Birth weight was $2300 \mathrm{~g}(-2.25 \mathrm{SD})$, length was $45 \mathrm{~cm}(-1.9$ $\mathrm{SD})$, and head circumference was $32.5 \mathrm{~cm}(-0.7 \mathrm{SD})$. Cutis laxa and prominent veins in the scalp and abdominal wall attracted medical attention in infancy, but a skin biopsy was not contributory. Psychomotor development was mildly retarded, with the infant showing head control at 6 months, walking alone at 20 months, and speaking meaning words at 24 months. Deciduous teeth were reported to have erupted at 2 years of age. The lack of eruptions of some permanent teeth in the upper jaw at 13 years of age was the basis for his referral to our hospital for further evaluation. His four maxillary permanent teeth of central and lateral incisors did not erupt. He had also a follicular cyst around the anterior teeth, necessitating sur-
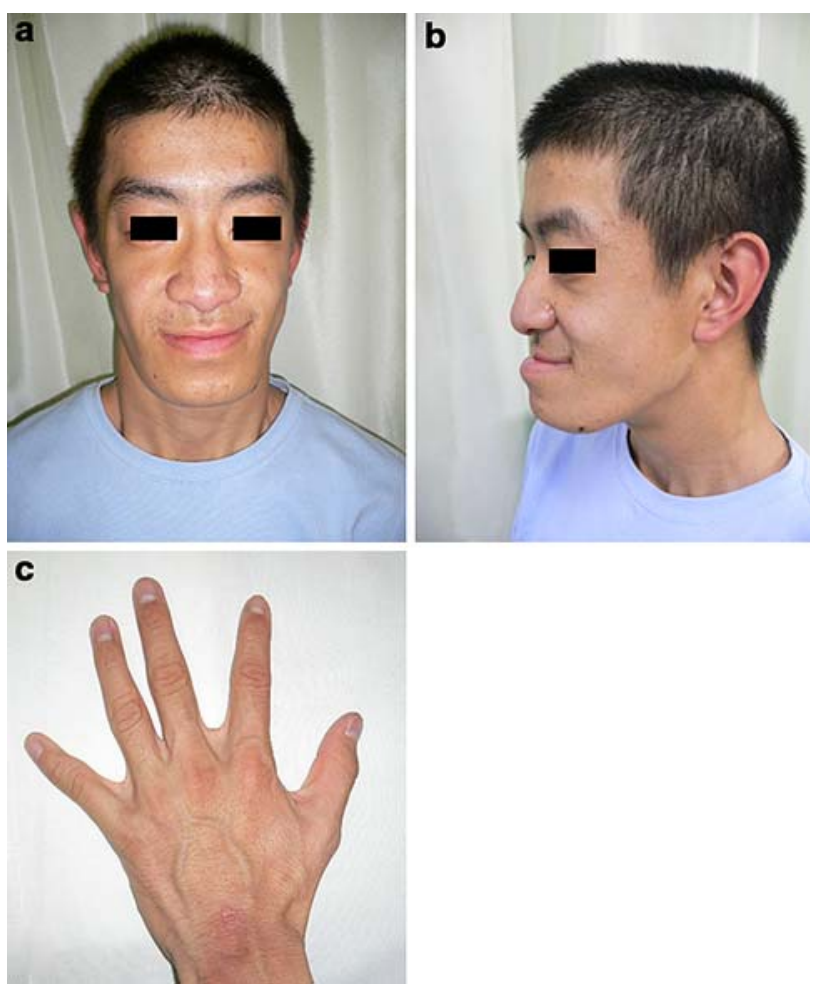

Fig. 1 Clinical photographs at 17 years of age. a, b A progeroid facial appearance with sparse hair, large auricles, and prognathism. c Interdigital webbing

gical extirpation. Enamel dysplasia was histologically confirmed. He showed a distinctive, progeroid facial appearance with sparse hair, large auricles, maxillar hypoplasia, high-arched palate, and prognathism (Fig. 1a,

Fig. 2 Radiological findings at 17 years of age.

a, b Hyperostosis of the calvaria, skull base and mandible. c Broad clavicles (arrows) and ribs, and sclerosis of metaphyseal equivalents of the spine. d Undermodeling of the short tubular bones and sclerosis of the carpal bones. e Metaepiphyseal sclerosis of the distal humerus, proximal ulna, and proximal radius. f Sclerosis of the sacrum, acetabulum, and proximal femur. g Sclerotic striations in the distal femur and proximal tibia
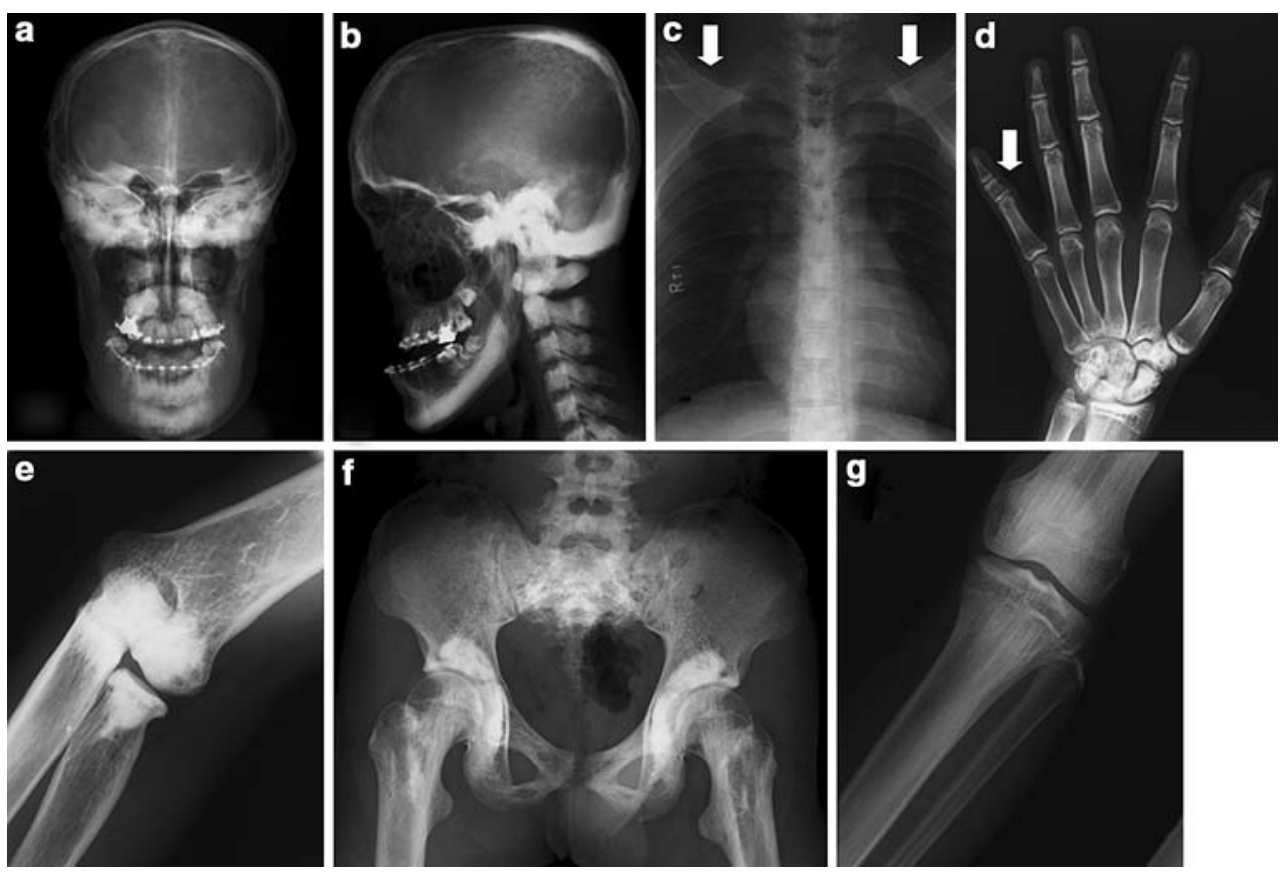


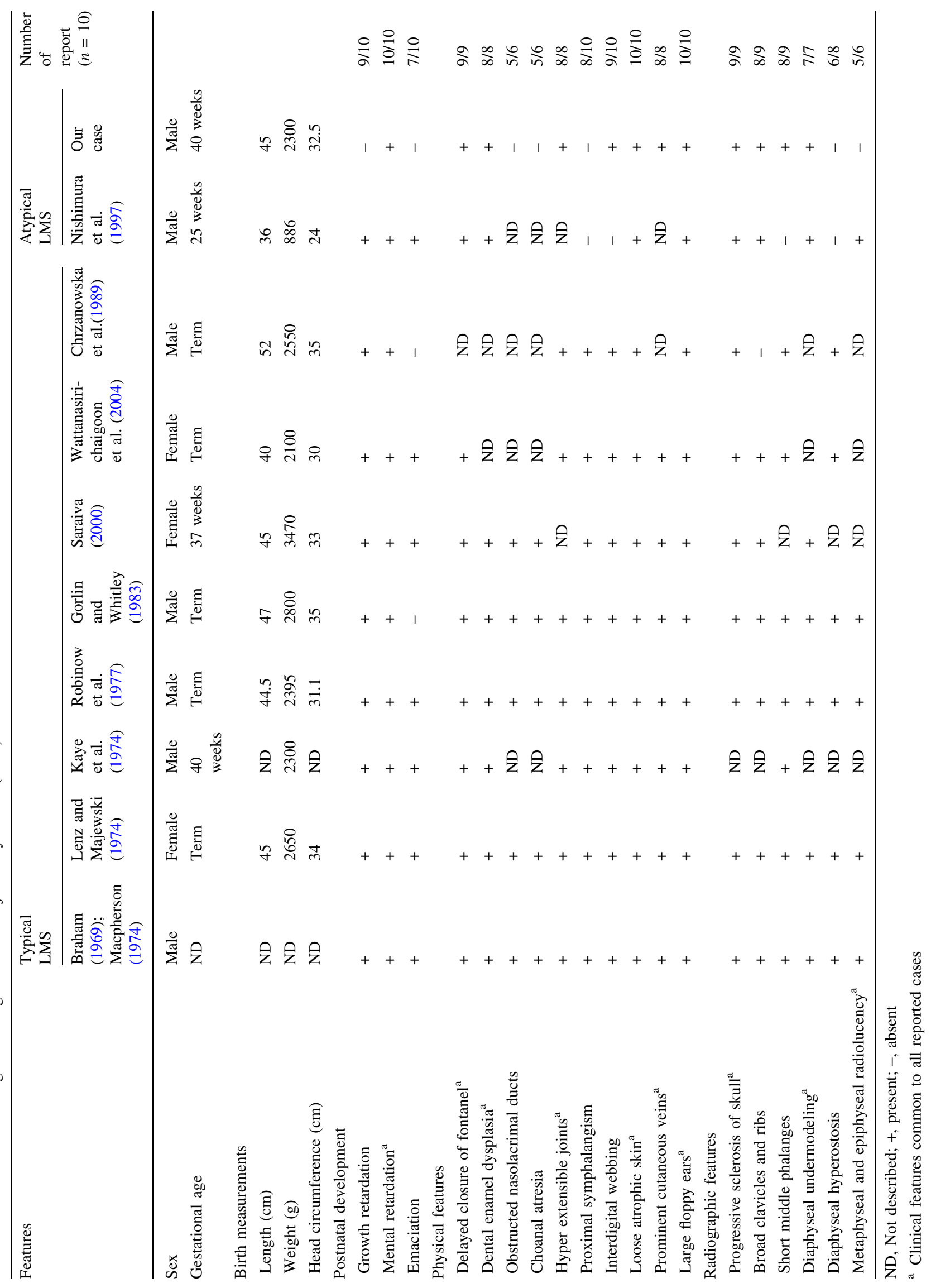


b). He had inter-digital webbing (Fig. 1c) and mild cubitus valgus. The skin was wrinkled and loose, and prominent veins were noted in the abdominal wall. $\mathrm{He}$ had mild mental retardation with a total intelligence quotient of 60 . Radiological examination showed cranial hyperostosis, particularly of the skull base, a sclerotic, thick mandible, broad clavicles and ribs, and diaphyseal undermodeling of the tubular bones. Diaphyseal hyperostosis was not evident, while epimetaphyseal sclerosis or longitudinal sclerotic striation was found in the long bones. Metaphyseal equivalents of the axial skeleton showed dense sclerosis (Fig. 2a-g). Bone scintigraphy showed an increased accumulation of radiopharmaceuticals, corresponding with sclerotic regions on the roentgenograms. During follow-up to 17 years of age, he showed a stable clinical course. At 17 years of age, he had a muscular build, a height of $164.5 \mathrm{~cm}(-1.0 \mathrm{SD})$ and a head circumference of $54 \mathrm{~cm}$ $(-1.3 \mathrm{SD})$ and weighed $56.2 \mathrm{~kg}(-0.54 \mathrm{SD})$. He underwent a surgical correction for severe anterior occlusion with prognathism. Mutation analyses for the transforming growth factor-beta1 gene (TGFB1) and the LDL receptorrelated protein 5 gene (LRP5) yielded normal findings.

\section{Discussion}

We describe a boy with a disorder that is classifiable into a group of sclerosing bone dysplasias. The disorder was radiologically characterized by cranial hyperostosis, metaepiphyseal sclerosis, and diaphyseal undermodeling. In addition, it was uniquely associated with cutis laxa, enamel dysplasia, and mental retardation. According to the 'Nosology and Classification of Genetic Skeletal Disorders: 2006 revision' (Superti-Furga and Unger 2007), sclerosing bone dysplasias are subclassified into three groups: (1) neonatal osteosclerotic dysplasia group, (2) increased bone density group (without modification of bone shape), and (3) increased bone density group with metaphyseal and/or diaphyseal involvement. A few disorders in the third group, such as Camurati-Engelmann disease, craniometaphyseal dysplasia, craniodiaphyseal dysplasia, and endosteal hyperostosis, share some radiological features with the present disorder. However, the unique constellation of skeletal, skin, dental, and mental abnormalities in the present disorder excludes the possibility of these disorders. In fact, molecular analyses for TGFB1 (the gene of Camurati-Engelmann disease) and LRP5 (the gene of endosteal hyperostosis, Worth type) were negative.

In the differential diagnosis, LMS deserves to be discussed. The disorder is characterized by craniodiaphyseal hyperostosis and brachymesophalangy with proximal symphalangism. It is complicated by mental retardation, somatic growth failure, cutis laxa, enamel hypoplasia, and webbed fingers and toes. The manifestations of previously reported LMS patients, LMS-like patients, and the present patient are summarized in Table 1. Most findings in LMS were shared by the present disorder. However, the differences include the absence of somatic growth failure and brachymesophalangy with proximal symphalangism in the present patient. LMS patients typically exhibit diaphyseal hyperostosis and metaepiphyseal radiolucency, while the present patient had only diaphyseal undermodeling and showed metaepiphyseal sclerosis. However, the degree of diaphyseal hyperostosis is described to vary considerably among LMS patients, and some patients with metaepiphyseal sclerosis are reported in the literature (Chrzanowska et al. 1989; Spranger et al. 2002). Thus, it is tempting to assume that the present disorder may fall at the mildest end in the phenotypic continuum of LMS, suggesting that the clinical spectrum of the disorder may be broader than currently thought.

\section{References}

Braham RL (1969) Multiple congenital abnormalities with diaphyseal dysplasia (Camurati-Engelmann's syndrome). Oral Surg 27:20 26

Chrzanowska KH, Fryns JP, Krajewska M, Van den Berghe H, Wisniewski L (1989) Skeletal dysplasia syndrome with progeroid appearance, characteristic facial and limb anomalies, multiple synostoses, and distinct skeletal changes: a variant example of the Lenz-Majewski syndrome. Am J Med Genet 32:470-474

Gorlin RJ, Whitley CB (1983) Lenz-Majewski syndrome. Radiology 149:129-131

Kaye CI, Fisher DE, Esterly NB (1974) Cutis laxa, skeletal anomalies, and ambiguous genitalia. Am J Dis Child 127:115-117

Lenz W, Majewski F (1974) A generalized disorder of the connective tissues with progeria, choanal atresia, symphalangism, hypoplasia of dentine and craniodiaphysial hypostosis. Birth Defects Orig Arctic Ser 10:133-136

Macpherson RI (1974) Craniaodiaphyseal dysplasia, a disease or group of diseases? J Can Assoc Radiol 25:22-33

Majewski F (2000) Lenz-Majewski hyperostotic dwarfism: reexamination of the original patient. Am J Med Genet 93:335-338

Nishimura G, Harigaya A, Kuwashima M, Kuwashima S (1997) Craniotubular dysplasia with severe postnatal growth retardation, mental retardation, ectodermal dysplasia, and loose skin: Lenz-Majewski-like syndrome. Am J Med Genet 71:87-92

Robinow M, Johanson AJ, Smith TH (1977) The Lenz-Majewski hyperostotic dwarfism. J Pediatr 91:417-421

Saraiva JM (2000) Dysgenesis of corpus callosum in Lenz-Majewski hyperostotic dwarfism. Am J Med Genet 91:200-202

Superti-Furga A, Unger S (2007) Nosology and classification of genetic skeletal disorders: 2006 revision. Am J Med Genet 143A:1-18

Spranger JW, Brill PW, Poznanski A (2002) Bone dysplasias: an atlas of genetic disorders of skeletal development, 2nd edn. Oxford University Press, Oxford

Wattanasirichaigoon D, Visudtibhan A, Jaovisidha S, Laothamatas J, Chunharas A (2004) Expanding the phenotypic spectrum of Lenz-Majewski syndrome: facial palsy, cleft palate and hydrocephalus. Clin Dysmorph 13:137-142 